\title{
New Water-Soluble Photoresists Utilizing Acrolein Modified Polyvinylalcohol
}

\author{
Nobuaki Hayashi, Hajime Morishita, Masato Ito, \\ Kyoko Kojima and Tetsuo Suzuki \\ Electron Tube and Devices Division, Hitachi Ltd., \\ Kokubunji, Tokyo 185, Japan
}

The acrolein modified PVA(APVA) was prepared by Michael additon of acrolein with PVA. The new water soluble photoresist that consists of APVA and sodium dichromate(SDC) shows higher sensitivity than that of conventional PVA-SDC photoresist.

\section{Introduction}

The water-soluble photoresists have been widely used in the production of a phosphor screen and a black matrix(BM) of a color TV tube. Many of these photoresists comprise water-soluble polymers and dichromate or azide compounds as sensitizers.

In manufacturing the large-sized color TV tubes, the problem is that the illumination intensity on an exposed film decreases because of the faceplate to be exposed is apart from a light source, therefore the exposure time becomes long. For these reason, high sensitivity have been required for high throughput. Many kinds of photoresists have been reported for these purpose. PVA and dichromate system is one of the oldest resist applying for industrial use. They are still using in phosphor screening, because of dispersing ability for phosphor powder.

In this paper, we will report on the new water-soluble photoresist consisting acrolein modified PVA and dichromate. 


\section{Experimental}

\section{Synthesis of Acrolein Modified PVA(APVA)}

Acrolein modified PVA was synthesized by the method shown in Fig. 1. One kilogram of $10 \%$ PVA(Kuraray, Poval-224) aqueous solution was stirred in 2 litter flask with reflux condenser, thermometer, and dropping funnel. Acrolein $(3.2 \mathrm{ml})$ was added dropwise at room temperature. Then the solution was stirred and heated at $60^{\circ} \mathrm{C}$ for 12 hours. The reaction mixture was cooled and poured into a large amount of acetone. Precipitate was collected and dried under vacuum. The degree of condensation was determined from the UV absorption in aqueous solution utilizing 3ethoxypropionaldehyde (molar absorption coefficient; $\varepsilon=41 \mathrm{~L} / \mathrm{mol}^{*} \mathrm{~cm}$ at $267 \mathrm{~nm}$ ) as the model compound.

\section{Characterization of Materials}

UV absorption spectra were measured with a Hitachi U-3410 spectrophotometer. Infrared spectra were recorded on a Perkin-Elmer 1720 FTIR spectrophotometer using thin films spin coated on aluminum-deposited glass substrates.

\section{Evaluation of photoresists}

The composition of photoresists in this study were shown in Table 1. Sodium dichromate(SDC) is used as photosensitizer in this study.

Table 1. Composition of Photoresists

\begin{tabular}{|c|c|c|}
\hline Material & New resist & Conventional resist \\
\hline SDC & $0.14 \mathrm{wt} \%$ & 0.14 \\
\hline APVA & 2 & 0 \\
\hline PVA & 0 & 2 \\
\hline water & residual part & residual part \\
\hline
\end{tabular}

The photoresists were spread on a glass plate by spinning, and dried by a infrared lamp. The thickness of the films were measured by an Alpha-Step 200 (TENCOR Instruments) profilometer. The film was exposed using a 500 W ultra-high pressure mercury lamp through $365 \mathrm{~nm}$-band pass filter. The intensity of the light used to irradiate a photoresist film was measured by a calibrated thermocouple. After the film was developed in water and dried, the remaining film thickness at the exposed areas were measured. The gel dose (sensitivity) was found by extrapolating the gel curve to zero film thickness. The quantum yield of insolubilization in a photoresist was calculated from the gel dose $\mathrm{Eg}\left(\right.$ einstein $/ \mathrm{cm}^{2}$ ) by the expression 


$$
\Phi_{\imath}=\frac{\mathrm{rd}}{\mathrm{AE}_{2} \mathrm{M}_{*}}
$$

where $r$ is the thickness of a film(cm), $d$ is the density of a film, $A$ is the fraction of incident photons absorbed by the phtosensitizer in a film, and $\mathrm{Mw}$ is the weight-average molecular weight of PVA. The progress of the photoreduction of $\mathrm{Cr}(\mathrm{VI})$ in a film during irradiation was monitored by spectrometry with a UV-visible spectrometer. The quantum yield of photoreduction from $\mathrm{Cr}(\mathrm{VI})$ to $\mathrm{Cr}$ (III) was derived from the dependence of the rate of chemical conversion(dx/dt) on exposure dose, via the equation(eq 2)

$$
\Phi_{\mathbf{s}}=\frac{\mathrm{n}}{\mathrm{IA}} \frac{d x}{d t}
$$

where $\mathrm{n}$ is the number of moles of $\mathrm{Cr}(\mathrm{VI})$ in $1 \mathrm{~cm}^{2}$ of the film, I is the irradiation flux (einstein $/ \mathrm{cm}^{2} . \mathrm{sec}$.), and $\mathrm{A}$ is the fraction of incident photons absorbed by the photosensitizer.[1]

\section{Results and Discussion \\ Characterization of APVA}

New water soluble polymer was synthesized from PVA and acrolein at $60^{\circ} \mathrm{C}$ for 12 hours. IR, NMR and UV spectra of polymer were shown in Fig. 2, Fig. 3 and Fig. 4 respectively. IR and NMR spectra were almost same as the original PVA, but UV spectrum was different from the starting materials. As can be seen in Fig. 2, strong carbonyl bands are observed at $1720 \mathrm{~cm}^{-1}$. PVA has residual carbonyl group from vinyl acetate parts, this carbonyl band in Fig. 2 is not assigned to aldehyde or acetate.

It is well known that PVA and aldehyde forms acetal structure with acid catalyst. In this case, product has double bond as shown in Fig. 5. In Fig. 3, no peaks are seen from double bond protons. And also aldehyde proton dose not find in this spectrum. We assumed that no peak from aldehyde in Fig. 3 was caused by $\mathrm{H}-\mathrm{D}$ exchange in $\mathrm{D}_{2} \mathrm{O}$ solution. In order to certify the aldehyde structure, we have prepared the acrolein modified polymer using $100 \%$ saponified PVA instead of conventional 88\% saponified PVA. IR spectrum of this polymer is shown in Fig. 6. As can be seen in this figure, new carbonyl band from aldehyde is observed. In these examination, we found that new polymer from PVA and acrolein have aldehyde group on side chain derived from Michael addition not acetal condensation.

The relationship between viscosity and the degree of condensation was shown in Fig. 7. With increasing the condensation degree, viscosity of the polymer solution was increased. But these values were smaller than those of acetal polymer prepared from acid catalyzed reaction of acrolein with PVA. 


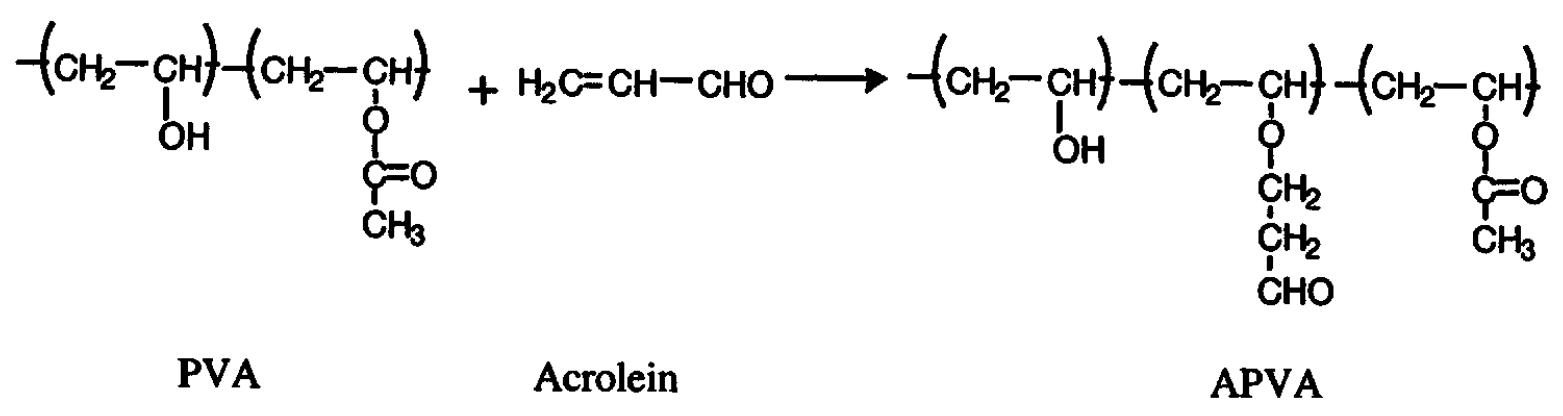

Fig 1 Synthetic Method of Acrolein Modified PVA

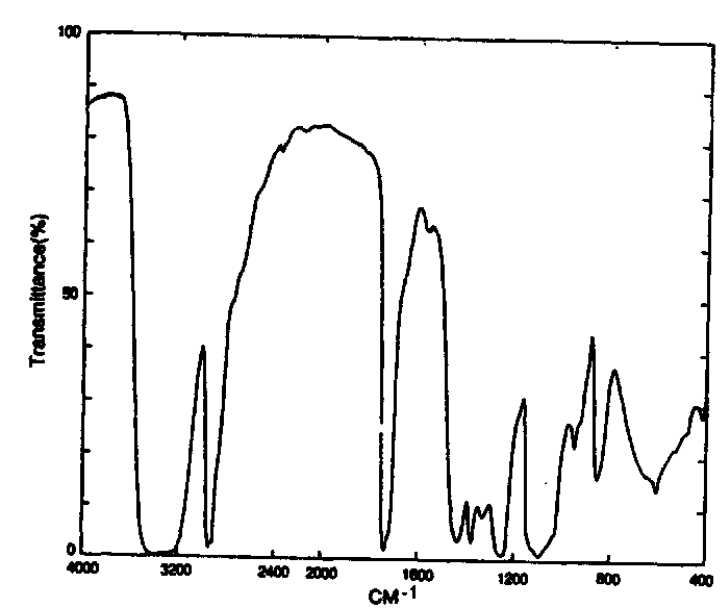

Fig 2 IR spectrum of APVA (acrolein 10 mol\%)

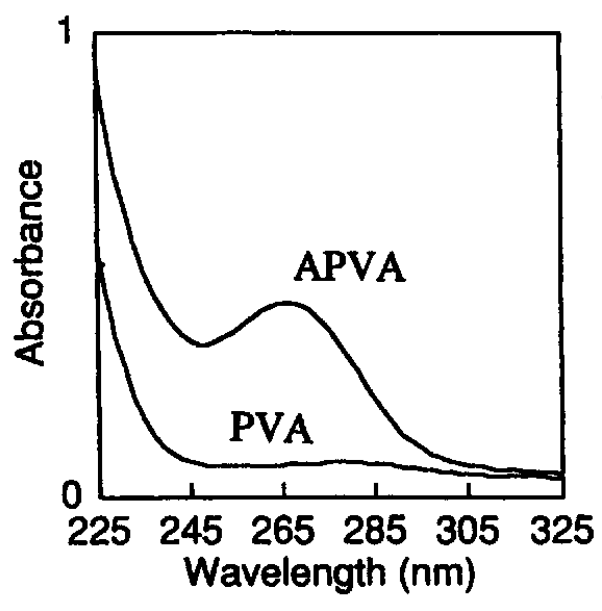

Fig 4 UV spectrum of APVA(acrolein $4 \mathrm{~mol} \%$ ) and PVA

$1.17 \mathrm{wt} \%$ aqueous solution

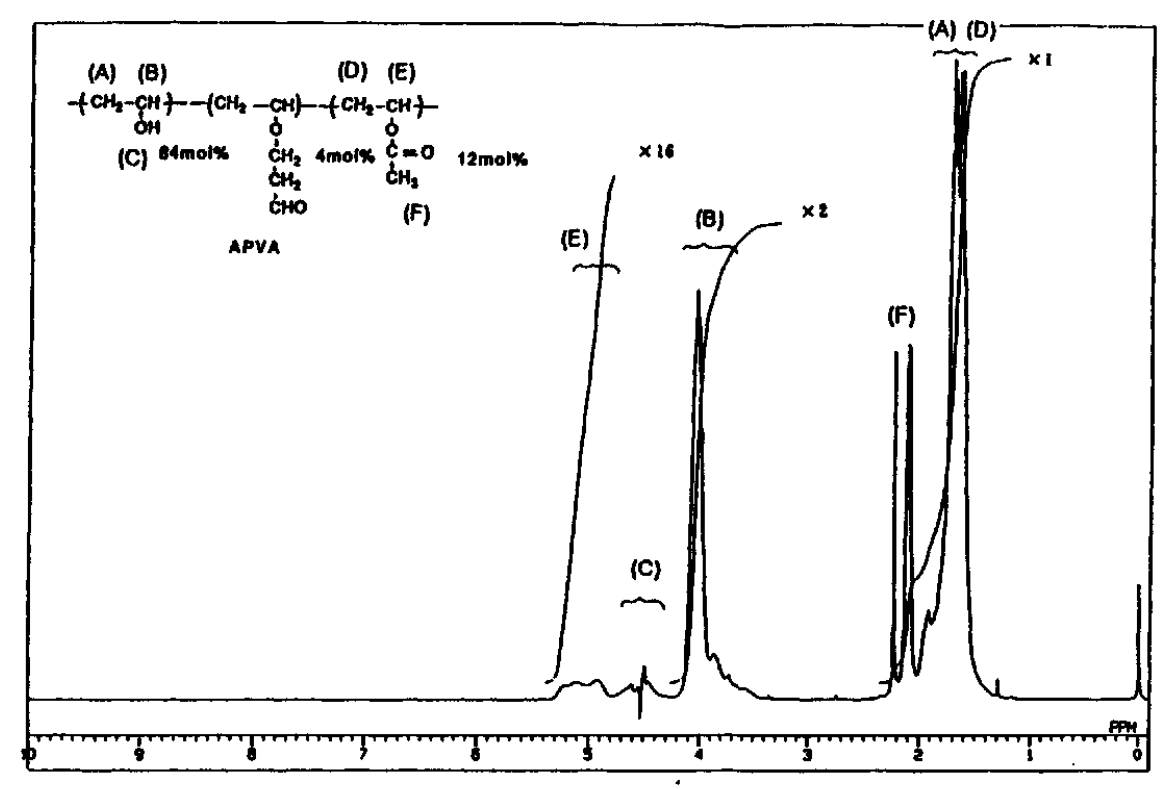

Fig 3 NMR spectrum of APVA(acrolein $4 \mathrm{~mol} \%$ ) $270 \mathrm{MHz} \mathrm{D}_{2} \mathrm{O}$ 


\section{Sensitivity of APVA-Dichromate Photoresist}

We have evaluated new water-soluble photoresist consisting of APVA and sodium dichromate (SDC) as a sensitizer. The sensitivity curves on APVA resist and conventional PVA-SDC resist were shown in Fig. 8. The sensitivity of APVA-SDC resist had 4 times higher than that of conventional PVA-SDC resist at 50\% film thickness remaining.

The relationship between sensitivity and degree of acrolein addition was shown in Fig. 9. The sensitivitys of resist film are dependent upon the content of aldehyde group. With increasing aldehyde group, the sensitivity of APVA-SDC resist increased, and then saturated over $4 \mathrm{~mol} \%$ addition of acrolein with PVA.

\section{Quantum Yield of Gel Formation}

The reaction mechanism of gel formation in PVA and SDC has been studied for a long time. [2]-[6] SDC sensitizer is existed acidic chromate anions, $\mathrm{HCrO}_{4}^{-}$, photosensitive species in PVA film. Only when reducing agents such as alcohol are presented, acid chromate anions is reduced to trivalent chromium spieces under UV irradiation. $\mathrm{Cr}$ (III) compound cross-linked to hydroxy group or carbonyl group on PVA. The new polymer APVA has aldehyde group, which is easily oxidized to carboxyloc acid, on side chain.

We have measured the quantum yield of gel formation $\left(\Phi_{g}\right)$ and reduction from $\operatorname{Cr}(\mathrm{VI})$ to $\operatorname{Cr}(\mathrm{III})(\Phi$, ). The results are shown in Table 2. As can be seen in Table 2, chromium compounds were easily reduced when they were exsited in the polymers containing alcoholic hydroxy group or reducing group such as aldehyde. Quantum yield of gelation varies much more than that of reduction of chromium compound in different polymers.

Table 2 Quantum yields of gel formation and reduction of chromium compound in polymer films

\begin{tabular}{|l|c|c|}
\hline polymer & $\Phi_{\mathrm{s}}$ & $\Phi_{\mathrm{g}}$ \\
\hline APVA & 0.57 & 0.16 \\
\hline PVA & 0.49 & 0.03 \\
\hline polyacrylamide & 0.25 & 0.002 \\
\hline PEG & 0.79 & 0 \\
\hline
\end{tabular}

We assume that high sensitivity in APVA-SDC resist comparing to PVA-SDC is caused by not only high quantum yield of reduction $\left(\Phi_{\mathrm{s}}\right)$, but also tendency in coordination to hydroxyl group or carbonyl group. 


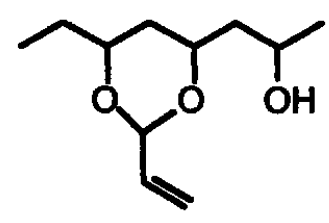

Fig 5 Structure of Modified PVA via acid catalyzed actal condensation

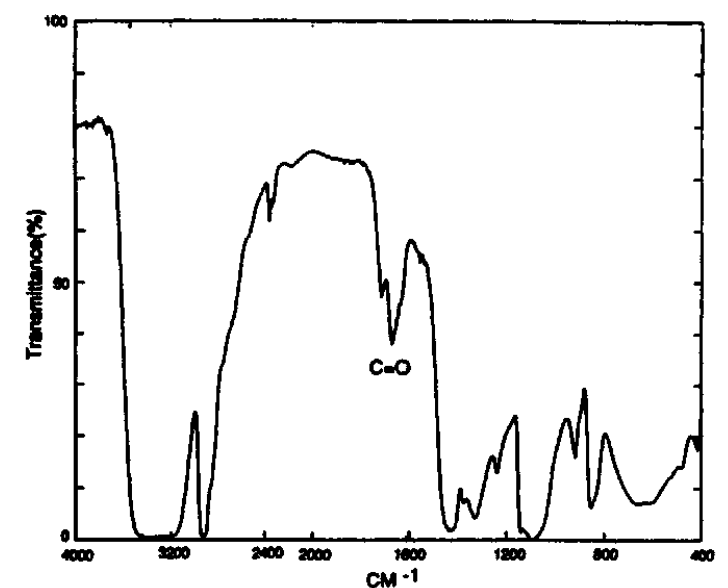

Fig 6 IR spectrum of APVA from 100\% saponified PVA

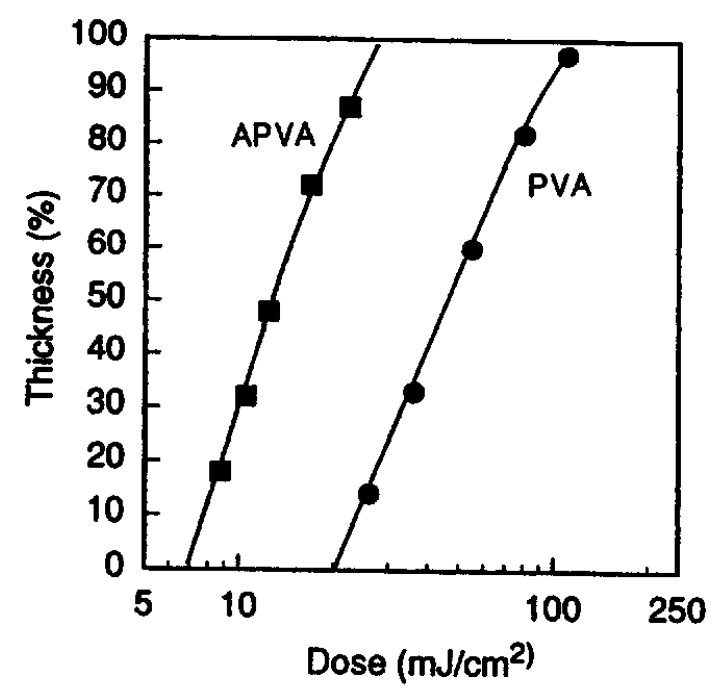

Fig 8 Sensitivity curves of APVA and PVA resist

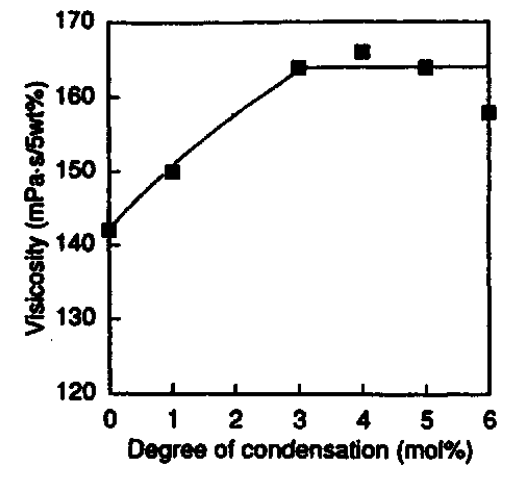

Fig7 Relationship between viscosity and degree of acrolein addition

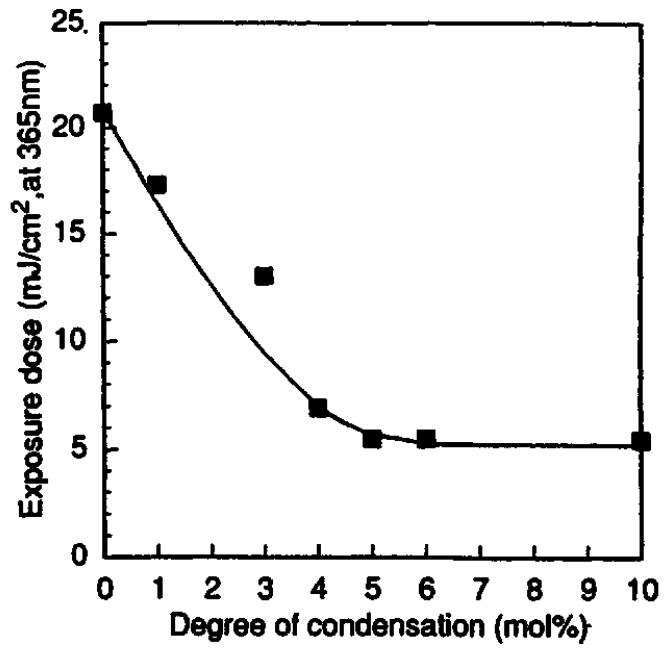

Fig 9 Relationship between sensitivity and degree of acrolein addition 


\section{Conclusion}

The acrolein modified PVA was prepared by Michael additon of acrolein with PVA. The new water soluble photoresist that consists of APVA and sodium dichromate shows higher sensitivity than that of conventional PVA-SDC photoresist. It was found that the increase in sensitivity did not depend only on to have a higher quantum yield of reduction from $\mathrm{Cr}$ (VI) to $\mathrm{Cr}$ (III) but also higher coordination ability of $\mathrm{Cr}$ (III) to carbonyl group.

\section{Acknowledgment}

The authors would like to thank Mr. Kazutoshi Terada and Mr. Hiroyuki Onishi of Kuraray Co. Ltd. for NMR analysis and their helpful discussions about PVA. They also thank Dr. Saburo Nonogaki, Mr. Masahiro Nishizawa, and Mr. Kiyoshi Miura for their helpful technical discussions.

\section{References}

1. F. H. Dill, et al, IEEE Transactions on Electron Devices, ED22, 445(1975)

2. M. Sasaki, et al, Kogyou Kagaku Zasshi, 70, 267(1967)

3. B. Duncalf, et al, J. Appl. Polymer Sci., 3, 1763(1964)

4. M. Bravar, et al, J. Polymer Sci., Symposium No. 40, 19(1973)

5. P. Datta, et al, Photo. Sci. Eng., 23, 203(1979)

6. L. Grimm, et al, J. Electrochem. Soc., 130, 1767(1983) 